\title{
Localisation of hyaluronan in the human intestinal wall
}

\author{
B Gerdin, R Hällgren
}

\begin{abstract}
By using biotin labelled proteoglycan core protein and an avidin enzyme system, hyaluronan (hyaluronic acid) was visualised in specimens of human jejunum. Intense staining for hyaluronan was seen in the loose connective tissue of the villi and of lamina propria while the epithelial layer was unstained. The muscularis mucosae showed only faint staining. The accumulation of hyaluronan in the subepithelial layer of the jejunal mucosa indicates that the previously reported high jejunal secretion of hyaluronan is due to passive diffusion from the subepithelial interstitium. The physicochemical characteristics conferred by hyaluronan may be important for the villi function.
\end{abstract}

The bowel wall contains many different cell types, including epithelial cells, smooth muscle cells, and vascular cells, capable of producing connective tissue components. The intestinal collagens consist mainly of type I and III collagens and are localised in the submucosa. ${ }^{1}$ The smooth muscle cells are considered to be the major producers of collagens in the intestine. ${ }^{12}$ Another important constituent of connective tissue is hyaluronan (hyaluronic acid by older nomenclature), which is a glycosaminoglycan composed of alternating units of glucuronic acid and $N$-acetylglucosamine. It is synthesised mainly by mesenchymal cells. It has recently been shown that the jejunal wall secretes con- siderable amounts of hyaluronan in health. Furthermore, jejunal secretion was increased in patients with Crohn's disease and coeliac disease. The appearance of high concentrations of hyaluronan in jejunal lumen in health and inflammatory bowel disease could not be attributed to a passive leakage from plasma but suggested a considerable local synthesis. In contrast, only minute amounts of procollagen III propeptide (PIIIP) were secreted into the jejunal lumen. The discrepancy indicated that either the turnover of hyaluronan was much higher than that of collagen type III, or that the structural localisation of hyaluronan was different. In this study we have visualised the localisation of hyaluronan in specimens of human intestinal wall by the use of a specific staining technique. ${ }^{45}$ The intense hyaluronan staining seen in the stroma of the villi suggests that the physicochemical characteristics conferred by hyaluronan are important for villi function.

\section{Methods}

\section{SPECIMENS}

Biopsy specimens were taken from the healthy jejunum in four patients in whom Roux-en $Y$ anastomoses had been performed after total gastrectomy. Specimens were fixed in an excess of $4 \%$ formalin with the addition of $1 \%$ cetylpyridinium chloride.

Accepted for publication 24 August 1990
(A) Hyaluronan specific staining of jejunal mucosa. A positive stain (brown) is seen in the mucosa, both in the villi and in the lamina propria, while lamina muscularis mucosae and the loose submucosa show only faint stains (counterstained with haematoxylin; original magnification $\times 100$ ).

\section{STAINING PROCEDURE}

An avidin enzyme, biotin protein system was used to detect hyaluronan in tissue. The system is based on the specific interaction between hyaluronan and the protein core of the cartilage proteoglycan. ${ }^{4}$ The technique entails three major steps: (i) the specific binding of biotin labelled protein to hyaluronan in the fixed tissue specimen; (ii) the binding of an avidin enzyme complex to the biotin; (iii) the visualisation of bound enzyme with a substrate.

The hyaluronan binding region of the cartilage proteoglycan (HABP) was prepared by affinity chromatography with HA-Sepharose,${ }^{6}$ and then linked to biotin as previously described. ${ }^{4}$ Tissue preparation and staining were performed as previously described. ${ }^{5}$ The specificity of the staining was checked with Streptomyces hyaluronidase (Seikagaku Fine Biochemicals, Tokyo, Japan). Control sections were incubated for four hours in a humidified chamber at $37^{\circ} \mathrm{C}$ with 500 units $/ \mathrm{ml}$ of hyaluronidase in $100 \mathrm{mM}$ sodium acetate buffer, $\mathrm{pH} 5 \cdot 8$. The sections were counterstained with haematoxylin. 


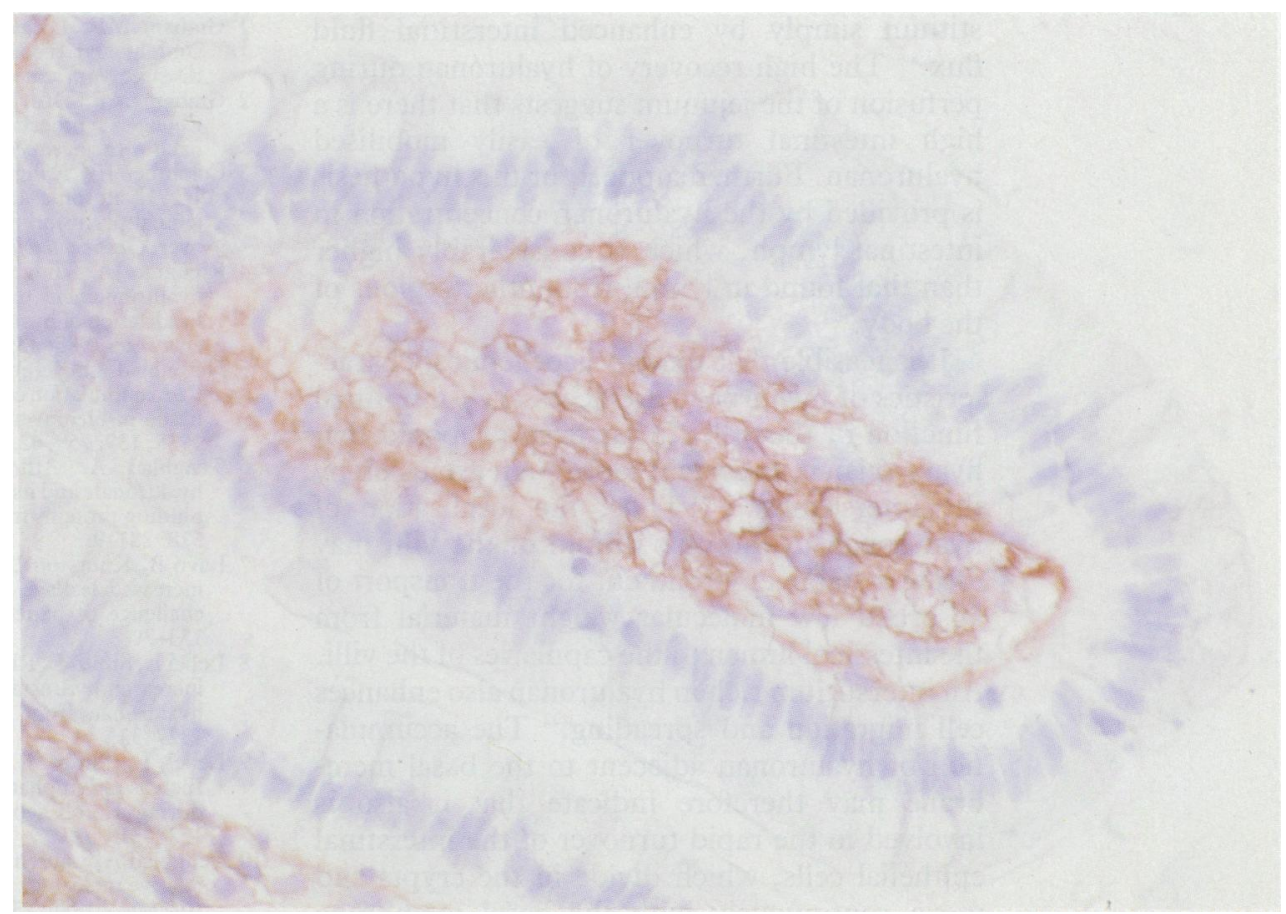

(B) Positive stain appears throughout the entire interstitial space, reaching the basal lamina of the epithelium and the villi capillaries. (Original magnification $\times 100$; oil immersion).

\section{Results}

In all specimens positive staining for hyaluronan was observed in the loose connective tissue of the villous and crypt area lamina propria (Figure A). The epithelial layer was unstained (Figure B) and there was only faint staining of the muscularis mucosae (Figure A). The loose connective tissue of the submucosa (Figure A) showed less intensive staining. The staining was most intense in the villi (Figure B). Hyaluronan occupied the entire interstitial space and a positive stain was seen from the basal membrane of the epithelium to the basal membrane of the villi capillaries. Transverse sections of the villi (Figure C) showed that the positively staining material was enriched subepithelially under the basal membrane. No staining for hyaluronan was seen between the epithelial cells. The specificity

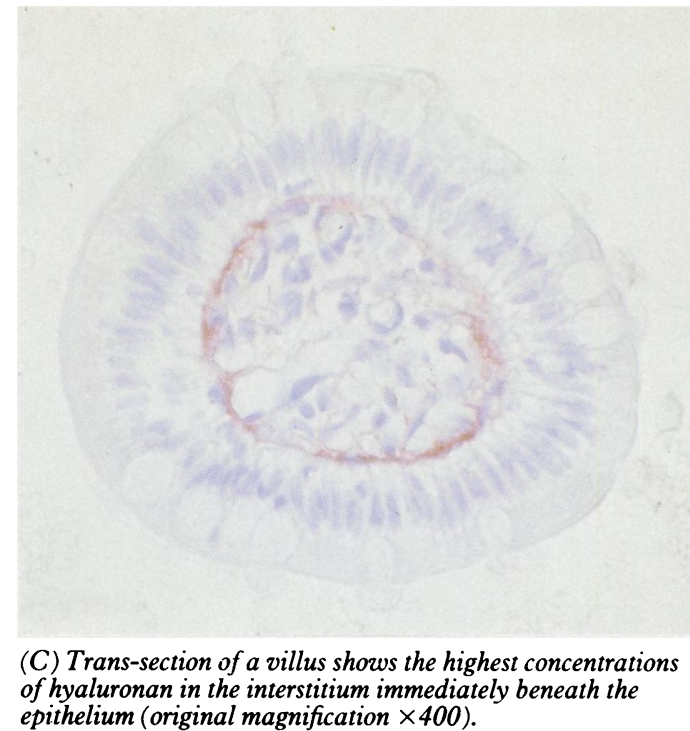

of the stain was verified after treatment of the sections with Streptomyces hyaluronidase.

\section{Discussion}

Previous studies on jejunal perfusion have indicated a considerable secretion of hyaluronan into the intestinal lumen. ${ }^{3}$ Hyaluronan has not hitherto been localised in intestinal tissue. Using biotin labelled proteoglycan core protein and an avidin enzyme system,${ }^{+5}$ however, we have now located considerable amounts of it in the connective tissue of the intestinal villi. Intensive staining for hyaluronan was seen especially under the epithelial basement membrane. These findings suggest that the appearance of hyaluronan in the jejunal lumen requires that it is translocated from the subepithelial interstitium to the lumen. If this is due to passive diffusion one would expect to find some hyaluronan also between epithelial cells. This was not the case. During the continuous turnover of the epithelial cells on the villous tips, however, the interstitium may be exposed to the intestinal lumen. Increased denuding of epithelial cells due, for example, to inflammatory events or toxic influences, may thus explain the increased jejunal fluid concentrations of hyaluronan found in patients with inflammatory bowel disease and in alcoholics. ${ }^{3}$ Although the rate of synthesis of hyaluronan may increase as a result of activation of the connective tissue of the villi in these conditions, the threefold increase in the luminal concentrations of hyaluronan within 30 minutes of gliadin challenge in the jejunum from patients with coeliac disease $^{7}$ should reflect increased mobilisation of interstitial hyaluronan rather than an enhanced local synthesis.

A considerable proportion of hyaluronan seems to be readily mobilised from the inter- 
stitium simply by enhanced interstitial fluid flux. ${ }^{89}$ The high recovery of hyaluronan during perfusion of the jejunum suggests that there is a high intestinal turnover of easily mobilised hyaluronan. Further support for this hypothesis is provided by the hyaluronan concentration in intestinal lymph, which is considerably higher than that found in lymph from other regions of the body. ${ }^{10}$

It is feasible that the physicochemical characteristics of hyaluronan are necessary for optimal function of the villi. It has been proposed that hyaluronan is important for protein exclusion by the interstitium. ${ }^{11-13}$ Thus, the localisation of hyaluronan in the interstitium of the villi may create a stable environment for the transport of absorbed low molecular weight material from the intestinal lumen to the capillaries of the villi. An interstitium rich in hyaluronan also enhances cell migration and spreading. ${ }^{14}$ The accumulation of hyaluronan adjacent to the basal membrane may therefore indicate that it also is involved in the rapid turnover of the interstinal epithelial cells, which divide in the crypts and move continuously with the basal membrane towards the tip of the villi. In previous studies hyaluronan has been found between the epithelial cells of the skin, suggesting a role for it in intercellular adhesion. ${ }^{15}$ We have not been able to find hyaluronan between the columnar epithelial cells of the intestine. Thus, the intercellular contacts between columnar epithelial cells seems to be qualitatively different from those between squamous epithelial cells.

The work was supported by grants from the Swedish Medical The work was supported by grants from the Swedish Medical Research Council, the Swacia Research Fund.
Pharmacis
1 Graham MF, Diegelmann RF, Elson CO, et al. Collagen content and types in the intestinal structures of Crohn's disease. Gastroenterology 1988; 94: 257-65.

2 Gram MF, Drucker DEM, Diegelmann RF, Elson CO. Collagen synthesis by human intestinal smooth muscle cells Collagen synthesis by human intestinal smo

3 Colombel JF, Hällgren R, Engström-Laurent A, Rambaud $\mathrm{JC}$. Hyaluronic acid and type III procollagen peptide in jejunal perfusion fluid as markers of connective tissue turn over. Gastroenterology 1989; 96: 68-73.

4 Ripellino JA, Klinger MM, Margolis RU, Margolis RK. The hyaluronic acid binding region as a specific probe for the localization of hyaluronic acid in tissue sections. $\mathcal{F}$ Histochem Cytochem 1985; 33: 1060-6.

5 Nettelbladt O, Bergh J, Schenholm M, Tengblad A, Hällgren R. Accumulation of hyaluronic acid in the alveolar interstitia tissue in bleomycin-induced alveolitis. Am Rev Respir Dis 1988; 139: 759-62.

6 Tengblad A. Affinity chromatography on immobilized hyaluronate and its application to the isolation of hyaluronate hyaluronate and its application to the isolation of hyaluronate
binding proteins from cartilage. Biochim Biophys Acta 1979; binding protein $281-9$.

7 Lavö B, Knutsson L, Lööf, Odlind B, Hällgren R. Signs of increased leakage from the jejunal mucosa during gliadin challenge of patients with coeliac disease. Gut 1990; 31: 153-7.

8 Lebel L, Smith L, Risberg B, Gerdin B, Laurent TC. Effect of increased hydrostatic pressure on lymphatic elimination of hyaluronan from sheep lung. $f$ Appl Physiol 1988; 64 1327-32.

9 Lebel L, Smith L, Risberg B, Laurent TC, Gerdin B. Increased lymphatic elimination of interstitial hyaluronan during E coli sepsis in sheep. Am $\mathcal{F}$ Physiol 1989; 256: during $\mathrm{E}$.

10 Tengblad A, Laurent UBG, Lilia K, et al. Concentration and relative molecular mass of hyaluronate in lymph and blood. relative molecular mass of hy
Biochem $\mathcal{F}$ 1986; 236: $521-5$.

11 Laurent TC. Structure, function and turnover of the extracellular matrix. Advances in Microcirculation 1987; 13: 15-34.

12 Comper WD, Laurent TC. Physiological function of connective tissue polysaccharides. Physiol Rev 1978; 58: 255-315.

13 Hällgren R, Gerdin B, Tufveson G. Hyaluronic acid accumula tion and redistribution in rejecting rat kidney graft; relationship to the transplantation edema. $\mathcal{F}$ Exp Med 1990; 171: 2063-76.

14 Toole BP. Glycosaminoglycans in morphogenesis. In: E D Hay, ed. Cellular biology of extracellular matrix. New York: Plenum, 1981: 251-94.

15 Tammi R, Ripellino JA, Margolis RU, Tammi M. Localization of epidermal hyaluronic acid using the hyaluronate binding region of cartilage proteoglycan as a specific probe. f Invest Dermatol 1988; 90: 412-4. 